\title{
Cartografías femeninas. Un acercamiento al mundo del trabajo con perspectiva de género. Córdoba, 1966-1976
}

\author{
[Female Cartographies. An Approach to the World of Work with a Gender \\ Perspective. Córdoba, 1966-1976]
}

\author{
Ana Laura Noguera \\ (Centro de Estudios Avanzados-FCS/Universidad Nacional de Córdoba, \\ Argentina) \\ analauranoguera@gmail.com
}

\begin{abstract}
Resumen
El presente trabajo se propone realizar un acercamiento inicial al mundo del trabajo cordobés en clave de género. Para ello utilizamos tres fuentes, muy disímiles entre sí, pero que nos permiten señalar algunos elementos centrales para comprender las características del empleo femenino en Córdoba en este periodo. ¿Qué tipo de actividad/trabajo realizaban las mujeres?, ¿qué complejidades, valoraciones sociales, tensiones y conflictos despertó su presencia en el mundo del trabajo cordobés? Considerando el periodo de intensa politización y radicalización, ¿cómo se vincularon las mujeres militantes del PRT-ERP y Montoneros con el mundo del trabajo?, ¿qué hacían?, ¿dónde trabajaban?

Partimos de una concepción que supone que tanto las experiencias de construcción de una clase social como las sexo-genéricas son producto de una interacción social dinámica, es decir, que están en permanente trasformación, siendo fundamental para el análisis la consideración de los diversos espacios y las distintas temporalidades.
\end{abstract}

Palabras claves: Género - Clase - Historia Reciente-Militancia.

\begin{abstract}
The present work intends to make an initial approach to the world of Córdoba's work in a gender key. For this, we use three sources, very different from each other, but that allows us to point out to some central elements to understand the characteristics of female employment in Córdoba during this period. What kind of activity/work did women do?, what complexities, social assessments, tensions, and conflicts did arouse its presence in the world of Córdoba's work? Considering the period of intense politicization and radicalization, how did the women militants of the PRT-ERP and Montoneros relate to the world of work? What did they do? Where did they work?

We start from a conception that assumes that both the experiences of building a social class and the sex-gender are the product of dynamic social interaction, that is, that they are in permanent transformation, being the consideration of various spaces and different temporalities fundamental in the analysis.
\end{abstract}

Keywords: Gender - Class - Recent History Militancy.

Recibido: 24/09/2019

Evaluación: 07/10/2019

Aceptado: 15/11/2019

Anuario de la Escuela de Historia Virtual - Año 10 - N 16 - 2019: pp. 137-152.

ISSN: 1853-7049

http://revistas.unc.edu.ar/index.php/anuariohistoria 


\section{Cartografías femeninas. Un acercamiento al mundo del trabajo con perspectiva de género. Córdoba, 1966-1976.}

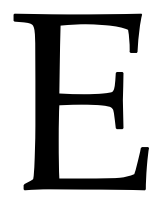

n 2019 se conmemoraron cincuenta años del denominado Cordobazo, ocurrido en mayo de 1969; se multiplicaron los actos, las jornadas académicas

y los homenajes. Al mismo tiempo, aparecieron numerosas publicaciones que referían al acontecimiento, así como también las que lo vinculaban con el ciclo de protestas que atravesó nuestro país a fines de la década del sesenta. Un recorrido por ellas da cuenta que mucho es lo que se ha dicho y escrito sobre este hecho histórico en particular y sobre la clase obrera -masculina- en general. Y si bien poco a poco observamos un reconocimiento respecto a la participación de las mujeres en los acontecimientos que tuvieron lugar durante toda la etapa, las figuras decisivas que se evocan para describirlos son mayoritariamente masculinas.

¿Qué sabemos sobre las mujeres obreras/trabajadoras en este período? Pregunta difícil de responder de manera certera en la medida en que todavía no hemos avanzado lo suficiente en investigaciones concretas que ayuden a reparar estas ausencias. Es conocida la dificultad que tienen las y los investigadores, preocupadas/os por esta época, de abordar la historia de las mujeres (y otrxs sujetxs) desde una perspectiva de género. $\mathrm{Su}$ ausencia en archivos y registros públicos o su presencia "a cuentagotas" en la prensa de la época contribuyen a esta invisibilización. Debe agregarse también la significativa gravitación de una perspectiva androcéntrica en la disciplina, aún en la actualidad.

Desde estas preocupaciones, el presente trabajo se propone realizar, a través de tres "instantáneas" -a modo de fotografías-, un acercamiento inicial al mundo del trabajo cordobés en clave femenina. ¿Cómo participaron las mujeres del mercado de trabajo?, ¿qué tipo de actividad/trabajo realizaban?, ¿qué complejidades, valoraciones sociales, tensiones y conflictos despertó la presencia de las mujeres en el mundo del trabajo cordobés? Considerando el periodo de intensa politización y radicalización, ¿cómo se vincularon las mujeres militantes del PRT-ERP y Montoneros con el mundo del trabajo?, ¿qué hacían?, ¿dónde trabajaban?

Para intentar responder algunos aspectos de estas preguntas analizaremos, en primer lugar, un informe que escribió en 1973 el Lic. Elias Baracat -director de Planeamiento de la provincia de Córdoba- titulado Situación de la mujer que trabaja, en la provincia de Córdoba. Como veremos, en él se describe la distribución del empleo femenino y la división (hetero)sexual del trabajo presente en la provincia durante este periodo. En segundo lugar, abordaremos los trabajos presentados por la socióloga Eva Chamorro Greca en el VIII Curso de Temporada organizado por la Universidad Nacional de Córdoba en 1967. Allí observaremos, a través de la mirada de una cientista social de la época, algunas valoraciones, tensiones y conflictos respecto de la presencia de mujeres 
en el mundo del trabajo. Finalmente, en un periodo marcado por la radicalización política de las y los jóvenes, retomaremos la Nómina de personas de Córdoba desaparecidas y asesinadas en los '70, elaborada por un equipo de investigación radicado en el Centro de Conservación y Documentación Audiovisual (CDA) de la Universidad Nacional de Córdoba para responder a la pregunta ¿dónde trabajaban las y los militantes del PRTERP y Montoneros?

Si bien las fuentes utilizadas son disímiles entre sí, el abordaje de las mismas permite establecer algunas pistas, a modo de primer ensayo, al mundo del trabajo con perspectiva de género. Partimos de una concepción que supone que tanto las experiencias de construcción de una clase social como las sexo-genéricas son producto de una interacción social dinámica, es decir, que están en permanente trasformación, siendo fundamental para el análisis la consideración de los diversos espacios y las distintas temporalidades. Por ello, el análisis hace foco en un espacio regional específico -Córdoba- con el objetivo no solo de aportar a su historiografía reciente, sino también como un estímulo para interpelar, tensionar, mostrar matices, complejidades y particularidades, es decir, establecer un diálogo entre lo nacional y lo local, entendiendo que se trata de un entramado complejo que conforma una determinada configuración sociohistórica (Jensen, 2010; Bohoslavsky, 2018).

\section{Notas en torno a género y clase en la historia reciente argentina}

En los últimos años, los estudios sobre la historia reciente han transformado paulatinamente el campo historiográfico argentino, promoviendo el uso de nuevas perspectivas analíticas y metodologías de trabajo (Águila, Luciani, Seminara y Viano, 2018; Franco y Levin, 2007). Las investigaciones sobre las décadas del sesenta y setenta desde una perspectiva de género no fueron ajenas a esta renovación; paulatinamente encontramos estudios que rescatan a las mujeres como sujetos sociales y problematizan críticamente las relaciones entre los géneros en diversos ámbitos. ${ }^{1}$ Sin embargo, solo una pequeña cantidad de trabajos indagó cómo estas transformaciones atravesaron y afectaron las experiencias de sociabilidad entre los sexos en el mundo del trabajo y (re)configuraron las representaciones construidas en torno a la mujer trabajadora.

Siguiendo la clásica caracterización de E. P. Thompson, la clase obrera se constituye como tal al verse a sí misma como un grupo con intereses y experiencias comunes (heredadas o compartidas), que ha construido un sentido de identidad colectiva y lucha compartida por oposición a los de otras clases. Sin embargo, tal como señala Catherine

\footnotetext{
${ }^{1}$ Entre ellos, podemos mencionar Andújar, et al. (2005; 2009 y 2010). Una diversidad de trabajos han examinado la participación política de las mujeres en las organizaciones político-militares, principalmente Montoneros y el PRT-ERP, como lo denotan, entre otras producciones, Grammático (2011); Martínez (2009); Noguera (2019); Oberti (2015); Pasquali (2008); Sepúlveda (2015) y Viano (2011). También encontramos investigaciones que indagan sobre los cambios socio-culturales del período (entre otros, Cosse, 2010; Cosse, Felitti y Manzano, 2010; Manzano, 2018) y sobre los grupos feministas y la diversidad sexual en la época (entre otros, D'Antonio, 2015; Felitti, 2006).
}

Anuario de la Escuela de Historia Virtual - Año 10 - N 16 - 2019: pp. 137-152. ISSN: 1853-7049 
140 | Cartografías femeninas. Un acercamiento al mundo del trabajo...

Hall, esta teorización sobre la emergencia de la clase trabajadora y del trabajador como sujeto político, presentada como esencialmente neutral en términos de género, en realidad siempre está articulada con un sujeto femenino o masculino (Hall, 2013). Hace tiempo que el feminismo, en sus diversas tendencias, realizó numerosas críticas a la categoría de clase social, siendo quizás uno de los pilares de ese señalamiento que no debe soslayarse el carácter sexuado de las experiencias de las y los sujetos (Hall, 2013; Fraser, 1997; Hartmann, 1980; Scott, 2011 [1999]; Federici, 2010). En este sentido, indagar la época desde la historia social del trabajo con perspectiva de género supone no solo considerar la experiencia de las y los sujetos y las relaciones sociales tejidas en términos materiales -siguiendo una perspectiva marxista-, fundamental sin dudas para la construcción de una identidad "clasista", sino también incorporar y problematizar desde el género tales construcciones sociales. Es decir, es necesario considerar los (al menos) dos elementos de una misma relación social, la clase generizada.

Incluir la dimensión genérica aporta, en primer lugar, a poner en tensión la división (hetero)sexual del trabajo, lo "productivo" y "reproductivo", las variadas formas y espacios que adquirió el trabajo, es decir, permite repensar los ámbitos públicos y privados. En segundo lugar, al poner en evidencia que las clases sociales están constituidas por sujetos sexuados, se incorpora al análisis las desigualdades y jerarquías entre los sexos, destacando diferencias aún dentro de la misma clase. En tercer lugar, desde este ángulo de análisis, se considera fundamental la recuperación de la agencia, de la dimensión política del género -y, por tanto, su vinculación con el ejercicio del podercomo un elemento primordial al momento de historiar las experiencias subjetivas de mujeres y varones. Recuperar sus acciones y cómo trasformaron (o no) las performances de género, complejiza de manera creciente los análisis enfocados solo en la subordinación y opresión femenina.

Asimismo, una cuestión central del aporte de la teoría del género a la historia social del trabajo es la incorporación de miradas más complejas sobre la sociabilidad y la cultura obrera. Se trata no solo de analizar las experiencias centradas en el sindicato o las fábricas, o las protestas en torno a diversas reivindicaciones, sino también qué redes de sociabilidad se establecieron en las distintas comunidades, el lugar de la familia en la conformación de esos vínculos, el uso del tiempo libre y de ocio, la cotidianeidad, el trabajo extra-hogareño, las representaciones construidas en torno a "lo masculino" y "lo femenino", todos elementos donde se conjugan fuertemente la clase con el género, ese doble antagonismo que habita y moldea los cuerpos.

En relación específicamente con las investigaciones que se enfocan en la intersección entre la historia social del trabajo y la perspectiva de género es importante decir, como ha señalado Andújar (2017), que existe en la pesquisa un predominio epocal de fines del siglo XIX hasta mediados del XX y una ausencia notable de trabajos que aborden esta 
temática con posterioridad al golpe de estado de $1955 .^{2}$ Este trabajo dialoga con estas producciones buscando explorar una problemática que se indexa a un campo en construcción -el del mundo del trabajo con perspectiva de género en las décadas de 1960 y 1970-, aún fragmentario y disperso, pero en el que van despuntando novedosas preguntas. En este sentido, resulta importante avanzar en la formulación de nuevos interrogantes y registros documentales, entendiendo la necesidad de profundizar en las relaciones sexo-genéricas en el mundo del trabajo en un contexto complejo signado por profundas trasformaciones políticas, sociales e intergenéricas.

\section{Instantánea primera. El informe Situación de la mujer que trabaja, en la provincia de Córdoba de Elias Baracat (1973)}

En 1973, el Lic. Elias Baracat -director de Planeamiento de la provincia de Córdobarealizó, a pedido de la Oficina Nacional de la Mujer, un informe titulado Situación de la mujer que trabaja, en la provincia de Córdoba. El mismo fue presentado en las Primeras Jornadas sobre trabajo de la mujer que se realizaron ese mismo año.

Antes de analizar algunos elementos presentes en el informe de Baracat, mencionaremos ciertos aspectos de la cartografía social cordobesa de mediados del siglo XX. Progresivamente, desde mediados de los años cincuenta, Córdoba fue transformando su fisonomía urbana y socio-económica convirtiéndose en una "nueva ciudad". La población de la capital aumentó, según los censos disponibles, de 586.015 en 1960 a 801.771 en solo una década, con un crecimiento intercensal del 16,7\% (INDEC, 1960; INDEC, 1970). Para entonces, la implantación de una progresiva industria automotriz y el crecimiento del sector industrial metal-mecánico -constituido en un polo “industrial moderno"-, modificó el desarrollo económico y urbanístico, transformando a la ciudad (y a la denominada "Gran Córdoba"), en la segunda concentración industrial del país después de Buenos Aires. Las industrias alimenticias, automotrices y químicas fueron las ramas más desarrolladas, llegando a ocupar a más de 10.000 personas. Por cierto, la clase obrera local no se limitaba a las industrias mecánicas. También incluía trabajadores calificados en los talleres ferroviarios de la ciudad, energía eléctrica y trabajadores gráficos junto a la gran masa de obreros no calificados en las demás ramas industriales (Brennan, 1996).

El boom industrial creó nuevas condiciones en el mercado de trabajo -centralmente para la mano de obra masculina-, permitiendo que la ciudad se convirtiera en un centro de atracción para nuevos trabajadores del interior, tanto de la provincia como del resto del país. Hacia 1970, el 35\% de la mano de obra estaba empleada en la industria, una

\footnotetext{
${ }^{2}$ Un trabajo nodal es el libro de Mirta Lobato (2007), Historia de las trabajadoras en Argentina (1869-1960). Sin embargo, el análisis se detiene iniciada la década de 1960. Por citar algunos de los trabajos que abordan una temporalidad como la aquí propuesta, pero focalizados en otros puntos del país, ver: Gatica (2009); Di Liscia, Lasalle y Lasalle (2011); Mitidieri (2014); Barragán Sáez (2015); Partenio (2017); D’Antonio (2018); Laitano y Nieto (2019).
}

Anuario de la Escuela de Historia Virtual - Año 10 - N 16 - 2019: pp. 137-152. ISSN: 1853-7049 
142 I Cartografías femeninas. Un acercamiento al mundo del trabajo...

cifra importante frente al 19\% ocupada en comercio, bancos y compañías de seguro, 35\% en servicios y $10 \%$ en ocupaciones diversas.

¿Qué ocurrió con el empleo femenino? Los datos disponibles para todo país señalan que hubo importantes transformaciones dentro del mercado de trabajo en relación al acceso y permanencia de las mujeres en el mismo. Si bien, como mencioné anteriormente, asistimos a un proceso de industrialización creciente, esto no impactó de manera significativa en la (re)distribución de la mano de obra femenina, que siguió ocupada mayormente en el sector servicios absorbiendo, junto con el sector comercio, el $85 \%$ del aumento total de la mano de obra femenina durante el período de mayor crecimiento (1960-1970) (Recchini de Lattes, 1980).

En cuanto al trabajo dentro del sector industrial/manufacturero, si bien se experimentó un descenso en la cantidad de trabajadoras en los rubros textiles y confecciones, continuó siendo mayoritario todavía en relación a las otras industrias. Los datos disponibles para todo el país indican que dentro de las industrias más dinámicas (automotriz, metalúrgica, fabricación de artefactos y productos eléctricos, productos químicos y petroquímica) la presencia de mujeres fue prácticamente mínima, solo el 1\% del crecimiento total de la mano de obra femenina durante el periodo 1947-1970. Aun así tuvo un crecimiento relativo importante, concentrándose principalmente en aquellas vinculadas a la electrónica y de armado de aparatos eléctricos -ligada sobre todo a la metalúrgica y construcción de transportes- y a las industrias químicas. Además, se registró una mayor participación de las mujeres en los departamentos administrativos de estas industrias. Dentro del sector terciario los servicios personales y de hogares continuaron siendo, según los datos del censo de 1970, la actividad que mayor proporción de empleo femenino concentró, constituyendo el $87 \%$ del empleo total de la misma (INDEC, 1970; Recchini de Lattes, 1980).

¿Qué pasaba en Córdoba con el empleo femenino? Como mencionamos anteriormente, en 1973 el Lic. Elias Baracat elaboró un informe titulado Situación de la mujer que trabaja, en la provincia de Córdoba. El escrito, de menos de 30 páginas, contiene una breve introducción y el resto está organizado en torno a tres tópicos: 1- Participación femenina en la estructura ocupacional de Córdoba. 2- Participación femenina en el sistema educacional. 3- Participación femenina en la ocupación del sector público provincial. Finalmente, varias páginas están destinadas a un anexo estadístico.

¿Qué nos dice el informe sobre las mujeres obreras/trabajadoras? En primer lugar, el autor señala que, al igual que en el resto del país, la mano de obra femenina representaba -en 1970- el 35,9\% dentro del total de la Población Económicamente Activa (PEA).

En segundo lugar, existía en el mercado laboral una marcada división sexual del trabajo, es decir, que el empleo femenino tenía una gran concentración en las actividades de servicios $(78,32 \%)$, especialmente en servicio doméstico, educación, comercio y sanitarios. Dentro del sector vinculado a educación podía observarse el alto porcentaje de mujeres ejerciendo la docencia en todos los niveles de enseñanza, siendo mayoría casi absoluta en los niveles inicial y primario (más del 90\%), decreciendo en las distintas 
modalidades del nivel medio (poco más del 60\%) y disminuyendo considerablemente en el nivel universitario (22,3\%). En tanto dentro del empleo público, el 59,2\% de los empleados provinciales eran varones, constituyendo el restante $40,8 \%$ las mujeres.

En el sector industrial su presencia era más bien escasa (el 16,6\%), y se concentraba principalmente en el rubro textil y de confección y en el sector calzado. Asimismo, dentro de las actividades realizadas por ellas más del $40 \%$ hacía tareas manuales no calificadas (a diferencia de los varones que constituyen un 23\%), descendiendo marcadamente su presencia en puestos de altos jefes, técnicos y profesionales.

En tercer lugar, a pesar de constituir un porcentaje mayor en el ítem "Nuevos Trabajadores" (el 2,91\% frente al 1,21\% de los varones), para 1970 la desocupación femenina en Córdoba era del 7,5\%, entre 2 y 4 veces mayor en relación a los varones, que tenían un porcentaje del 2,4\%.

Sin embargo, dentro de la PEA femenina solo el $29,2 \%$ trabajaba o buscaba trabajo a diferencia de la población masculina en que el 57,4\% trabajaba o buscaba trabajo. De 314.158, que constituía la población no económicamente activa de mujeres, el 46,4\% eran amas de casa (el restante niñas, estudiantes o pensionadas). Aunque las estadísticas no contemplen una multiplicidad de situaciones respecto al mercado laboral, de los datos aportados por Baracat se deduce que, pese al incremento de las mujeres en el mercado de trabajo, un porcentaje significativo no trabajaba fuera de su hogar y, las que lo hacían, se vinculaban con trabajos/profesiones ocupados históricamente por una abrumadora mayoría femenina.

\section{Instantánea Segunda. La Mujer-Madre que sale a trabajar: la perspectiva sociológica de Eva Chamorro Greca}

Hacia fines de los años sesenta, en 1967 más exactamente, la Universidad Nacional de Córdoba organizó el VIII Curso de Temporada titulado, en esa oportunidad, "La mujer argentina y latinoamericana". Dentro de un extenso programa, que incluyó las exposiciones de Alicia Moreau de Justo, Marina Waisman, entre otras/os disertantes, la socióloga Eva Chamorro Greca ${ }^{3}$ presentó dos trabajos: uno titulado "La mujer en la estructura familiar" y el otro denominado "La madre que sale a trabajar". Preocupada por la diversidad de perspectivas -a favor y en contra- del trabajo de las mujeres, la autora se proponía en esa oportunidad presentar algunas conclusiones de una investigación que llevó adelante a los fines de "examinar empíricamente los efectos del empleo y ocupación laboral extra hogareña de la madre" (Chamorro Greca, 1967a, p. 242).

Basando su análisis en noventa entrevistas/encuestas individuales, realizadas a mujeres con diferentes niveles ocupacionales, residentes en la ciudad de Córdoba,

\footnotetext{
${ }^{3}$ Eva Chamorro Greca nació en Córdoba, recibió el título de abogada en 1957 y se doctoró en Derecho y Ciencias Sociales en 1968. Perteneció, junto a Carlos Agulla y otros sociólogos, al Instituto de Sociología de la Facultad de Derecho de la UNC.
}

Anuario de la Escuela de Historia Virtual - Año 10 - N 16 - 2019: pp. 137-152. ISSN: 1853-7049 
144 | Cartografías femeninas. Un acercamiento al mundo del trabajo...

Chamorro observó distintas variables en relación a esta problemática. Presentado por la autora como un "problema" sociológico, justificaba esta caracterización en la medida en que la combinación de actividades vinculadas al ámbito familiar (cuidado de los hijos, de la casa y del esposo) -"porque por la división tradicional de funciones a ella le corresponde ser la ama de casa" - con actividades extra hogareñas generaban conflictos: "la mujer siente sobre sí un doble peso o una doble expectativa [...] O sea que este problema tiene una consecuencia individual y otra social" (Chamorro Greca, 1967b, p. 233).

En el análisis de Chamorro Greca se perciben los desplazamientos epocales respecto a los roles socialmente aceptados para cada género. Es decir, si bien se ubica a la mujer en la estructura social principalmente como "esposa y madre", es también una realidad del momento que ellas se han incorporado crecientemente al mercado del trabajo. Esto generaba tensiones y conflictos entre estas transformaciones y la persistencia de un modelo "tradicional" de domesticidad, que suponía que las mujeres debían cumplir también- con las actividades del hogar, el cuidado de los hijos y la cocina; además de las actividades profesionales, laborales (y por qué no, las políticas).

Sobre la base de estas consideraciones, el equipo de trabajo dirigido por la autora elaboró algunas hipótesis que nos parece interesante mencionar brevemente: 1- La industrialización lleva al cambio de estatus de los miembros de la familia, siendo el de la mujer el más afectado. Este cambio es debido a las nuevas posiciones ocupacionales y no a las tradicionalmente aceptadas como femeninas. 2- El status ocupacional de la esposa en tareas relacionadas a industria y comercio (tareas no realizadas antes) ha cambiado el esquema familiar a un modelo de igualdad entre padre y madre, que le dificulta al esposo mantener vestigios de patriarcalismo, ya que su mujer trabaja y contribuye con su salario. 3- La mujer no solo trabaja por necesidades económicas, sino porque le permite ejercer actividades que no realizan las mujeres que se dedican solo al hogar. 4- Esta nueva emancipación de la mujer no implica una asimilación a roles masculinos. Hay una segregación social para los nuevos estatus ocupacionales de la mujer, es decir, la ocupación la lleva a dedicarse a problemas que atañen principalmente al propio sexo. 5- Las actividades extra hogareñas han generado una aceptación de nuevos progresos técnicos para el hogar y no a la inversa (Chamorro Greca, 1967a, p. 244). ${ }^{4}$

Uno de los puntos de análisis sobre este "problema" (y los conflictos que generaba) era la colaboración del esposo en las tareas del hogar. Allí, señalaba que el 73\% de las esposas aceptaban que sus esposos ayudaran en el hogar "algunas veces", cuando ella estaba enferma, en caso de necesidad o por falta de servicio doméstico. Al entrecruzar otras variables como edad y escolaridad, resultaba que las madres jóvenes y las de escolaridad alta mencionaban en un $25 \%$ que sus esposos debían ayudar "siempre" o "a menudo". Las madres mayores y con otro tipo de escolaridad, se inclinaban por "algunas

\footnotetext{
${ }^{4}$ Para un análisis del impacto de la mecanización en el hogar en este periodo, ver: Pérez (2010).
} 
veces". En su exposición, la autora explicaba que las mujeres aceptaban la división de tareas en la casa como reflejo de la división del trabajo para varones y mujeres. Como conclusión, sostenía que la mujer madre que deja su hogar y sale a trabajar, vive "una dualidad de roles y siente sobre sí el peso de las valoraciones tradicionales y modernas" (Chamorro Greca, 1967a, p. 264).

\section{Instantánea Tercera. El Cordobazo y la militancia política de las mujeres ${ }^{5}$}

Durante los sesenta y setenta la participación política de las mujeres tomó un impulso arrollador, masivo. Los modelos revolucionarios a nivel internacional, principalmente Cuba y Vietnam, pero también el Mayo Francés y la Primavera de Praga, aparecieron como ejemplos a seguir para un importante número de jóvenes, que asumieron la lucha política y la militancia en pos de la construcción de una nueva sociedad, que se pensaba debía ser más justa.

El Cordobazo fue ciertamente una movilización mayoritariamente masculina. Esto fue así primeramente porque los sectores que la convocaron estaban constituidos en su mayoría por varones. Sin embargo, sería incorrecto decir que las mujeres no estuvieron presentes. Trabajadoras, obreras, estudiantes, todas se sintieron interpeladas por el hecho. Así, aunque en términos cuantitativos la movilización de mayo tuviera una impronta netamente masculina, también para las mujeres fue una bisagra en términos políticos. Para muchas fue su "bautismo de fuego".

En este sentido, el Cordobazo ejerció una influencia decisiva en el proceso de politización y radicalización, aumentando cuantitativamente la cantidad de activistas. De hecho, un observador de los sucesos de mayo de 1969, el sociólogo Juan Carlos Agulla, sostenía que, si bien las mujeres no representaban una gran cantidad, las mismas actuaron a la par de sus compañeros varones. Y que su presencia -constituida mayoritariamente por estudiantes-inauguraba la aparición de la militancia femenina en la vida social y política de la ciudad.

En este contexto, emergieron grupos y organizaciones revolucionarias que plantearon la opción de la lucha armada como instrumento de cambio y transformación. Montoneros -de filiación peronista- y el Partido Revolucionario de los Trabajadores (PRT), a través de su brazo armado, el Ejército Revolucionario del Pueblo (ERP), de orientación marxista/troskista, fueron dos de las organizaciones político-militares más numerosas. ¿Qué sabemos de las mujeres y los varones militantes de estas dos organizaciones en relación con el mundo laboral?, ¿qué hacían?, ¿dónde trabajaban?

Para acercarnos a esta problemática, utilizamos la Nómina de personas de Córdoba desaparecidas y asesinadas en los '70, elaborada por un grupo de investigación radicado en el Centro de Conservación y Documentación Audiovisual (CDA) de la Universidad Nacional de Córdoba, publicada por la UNC en 2016. La misma incluye a "todos aquellos

\footnotetext{
${ }^{5}$ Este apartado corresponde a un fragmento publicado en Noguera (2019). 
146 | Cartografías femeninas. Un acercamiento al mundo del trabajo...

que habiendo sido secuestrados, desaparecidos y asesinados dentro o fuera de la provincia de Córdoba hubiesen permanecido en ésta durante algún período entre los años '60 y '70" (Romano, 2016, p. 137).

$\mathrm{Si}$ bien solo analizamos las trayectorias de las mujeres $\mathrm{y}$ varones Detenidos/Desaparecidos/Asesinados (en adelante DDA) pertenecientes a las dos organizaciones antes mencionadas, consideramos que las conclusiones pueden servir también para caracterizar al conjunto de la militancia cordobesa. Al momento de su consulta, la nómina registra un total de 1.054 víctimas de la represión paraestatal y el terrorismo de Estado en Córdoba, de los cuales el 75\% son varones, correspondiendo el restante $25 \%$ a mujeres.

¿Reprodujeron las y los militantes los criterios de la división (hetero)sexual del trabajo? Como referimos anteriormente, siguiendo los datos proporcionados por el censo de 1970, el sector servicios, comercio y finanzas y seguros fue donde mayor concentración de trabajadoras hubo. Existió un aumento notable de mujeres en la administración pública y en el rubro servicios sociales y comunales. El $72 \%$ de la población económicamente activa femenina estaba ocupada en este último y comprendía enfermeras, maestras, profesoras, profesionales. Esto da cuenta de la estrecha relación entre las mejoras en el acceso de las mujeres a la educación formal y las posibilidades de inserción en el mercado de trabajo (INDEC, 1970; Recchini de Lattes, 1980, pp. 49-50).6

Los datos precedentes resultan significativos para el análisis de las ocupaciones de las mujeres militantes DDA. En primer lugar, encontramos una gran cantidad de docentes en todos los niveles de enseñanza. Aquellas que habían conseguido el título superior pertenecían mayoritariamente a las carreras de Humanidades y, en menor medida, a Medicina, Arquitectura, Servicio Social y Abogacía. Así, muchas de ellas se desempeñaron dentro de sus profesiones, tanto en instituciones públicas y/o de gobierno (hospitales, bibliotecas, secretarías y/o ministerios), como en privadas. También accedieron a puestos en la administración pública provincial y municipal independientemente de su formación educativa-, algunas fueron empleadas bancarias, vendedoras en comercios y secretarias/administrativas de oficina; desde allí, algunas se convirtieron en delegadas gremiales dentro de los sindicatos respectivos. Entre las trabajadoras "independientes" también hubo propietarias de pequeños negocios, modistas, peluqueras y vendedoras de cosméticos. Además, encontramos varias que se desempeñaban como "amas de casa".

Muchas de ellas transitaron por más de un empleo, algunos vinculados a sus profesiones y otros alejados de ellas. Según los testimonios de la época, estaba bien visto que las y los militantes se incorporaran a una actividad productiva. Ambas

\footnotetext{
${ }^{6}$ Hacia fines de los años sesenta, se experimentó un proceso de importante crecimiento de la matrícula universitaria al mismo tiempo que se asistió a una creciente feminización de la misma, es decir, a un aumento significativo de ingreso de mujeres. En la Universidad Nacional de Córdoba, por ejemplo, en 1968 se inscribieron 9.742 mujeres (y 17.108 varones) y para el año 1973 figuraban 20.506 (y 23.731 respectivamente); en los diez años que van de 1966 a 1976 creció en un 15,3\%, en relación a los varones. Ver: Noguera (2019).
} 
organizaciones alentaban la proletarización de sus miembros como un modo de afrontar "como experiencia" trabajos alejados de su cotidianeidad social.

Así, el empleo doméstico fue la opción laboral de varias. A lo mencionado anteriormente, se le sumaba lo beneficioso de la informalidad de la actividad, ya que para aquellas que militaban y estudiaban (y/o tenían hijos) era la manera de trabajar sin ataduras de horarios y las formalidades del empleo "en blanco". Además, fue la forma de subsistencia para quienes, o bien tenían antecedentes penales, o bien habían comenzado a ser perseguidas por las fuerzas de seguridad.

En lo que respecta al trabajo dentro de los sectores industriales, solo una pequeña proporción eran obreras. En Córdoba, las fábricas que empleaban mayoritariamente mano de obra femenina eran las del sector del calzado, textiles, vidrio, alimentación y en la producción de cables y componentes eléctricos para autos Renault en la planta de Industria Latinoamericana de Accesorios S.A. (ILASA).

Por su parte, a diferencia de las mujeres, una gran cantidad de los varones DDA pertenecían al mundo obrero. El desarrollo de los grandes complejos metal-mecánicos durante los sesenta y setenta favoreció el crecimiento de la mano de obra masculina en este sector. Más del 10\% de los represaliados eran trabajadores de las grandes fábricas asentadas en Córdoba en el período: Ika-Renault, IME, Fiat, Transax, Perkins, entre otras. Las escuelas técnicas proveyeron una formación particular y posibilitaron el ingreso de un importante número de jóvenes al mundo de las fábricas, lugar apreciado por los altos sueldos percibidos por los obreros de este sector. Desde allí, en el clima de movilización socio-política y radicalización, ingresaron a la militancia y posteriormente algunos se inscribieron en la universidad.

Por otra parte, y a la inversa, varios estudiantes ingresaron al ámbito obrero no solo como opción laboral, sino también con la finalidad de desarrollar el trabajo político en ese espacio, considerado potencialmente revolucionario, sobre todo, luego de los sucesos del Cordobazo. Otra industria que absorbió una significativa cantidad de mano de obra masculina fue el rubro construcción (albañiles, pintores, gasistas). También en este sector, la educación en las escuelas técnicas -específicamente el título de maestro mayor de obras- proporcionó una salida laboral inmediata para sus egresados. Al igual que el empleo doméstico para las mujeres, la línea política de las organizaciones alentaba a los militantes a tener experiencias laborales y habitacionales cercanas al "pueblo trabajador". Así, la informalidad del rubro construcción posibilitó a los militantes tener una mayor disponibilidad para participar o subsistir en épocas de persecución.

Dentro de las industrias de bienes de consumo, específicamente alimentación, se verifican varios varones represaliados, especialmente en dos espacios que destacaban por sus reiterados conflictos gremiales en el periodo considerado. Por un lado, la láctea SanCor y, por otro, el Frigorífico Mediterráneo.

En relación a los profesionales, se encuentran en la Nómina, médicos, ingenieros, abogados, arquitectos, agrónomos, contadores. Muchos de ellos se desempeñaron en instituciones públicas y/o privadas (hospitales, bancos, oficinas de gobierno) y algunos 
148 | Cartografías femeninas. Un acercamiento al mundo del trabajo...

pocos fueron docentes en distintos niveles de enseñanza. Otros trabajaban como empleados administrativos o de comercio, o bien tenían su propio negocio, eran viajantes, vendedores ambulantes, trabajadores independientes y periodistas (gráficos, camarógrafos, cronistas).

Numerosos varones fueron empleados de la administración pública nacional, provincial y municipal. En los datos analizados se observan trabajadores de Obras Sanitarias de la Nación, Ferrocarriles, Banco Nación y Provincia de Córdoba, Empresa Provincial de Energía de Córdoba (EPEC), Dirección Provincial de Vialidad (DPV), Correo, Empresa Nacional de Telecomunicaciones (ENTEL) y de las distintas reparticiones de la Municipalidad y del gobierno provincial. Es de destacar que muchos de ellos eran delegados gremiales o miembros de comisiones internas de fábrica, lo que da cuenta del carácter clasista de la violencia del terrorismo de Estado desde 1976 a 1983. Según los datos disponibles, el 30,2\% de los desaparecidos fueron obreros y, en el caso de provincias como Córdoba, con una fuerte presencia fabril, esa cifra ascendía a 41,90\% (Ortiz, 2019).

\section{Consideraciones finales}

En este trabajo, incipiente sin dudas, hemos intentado analizar algunos datos sobre el trabajo de las mujeres hacia fines de los años sesenta y principio de los setenta. Para ello, utilizamos tres fuentes, muy disímiles entre sí, pero que nos permiten poder señalar algunos elementos centrales para comprender las características del empleo femenino en Córdoba en este periodo.

En primer lugar, el informe de Baracat y los datos disponibles en los Censos, hacen suponer que es necesario establecer una distinción entre empleo femenino y masculino y matizar el impacto que tuvo el proceso de industrialización en el mismo. Es decir, que, si bien hubo significativas transformaciones dentro del mercado de trabajo en relación al acceso y permanencia de las mujeres, este proceso de industrialización no supuso una significativa (re)distribución de la mano de obra femenina, que siguió empleada en sectores "tradicionalmente" ocupados por ellas.

En segundo lugar, a través de las reflexiones que nos brindan los estudios realizados por Chamorro Greca, nos acercamos a un "problema social" de larga data: el trabajo femenino. Sin dudas, estas preocupaciones no eran novedosas. Al menos desde principio de siglo, cuando las mujeres se incorporaron crecientemente al mercado de trabajo, comenzaron a proliferar discursos a favor y en contra del mismo. Cada época le imprimió su propia dinámica y contenido específico, pero se mantuvo (y aún podemos vislumbrarlo en la actualidad) un eje conflictivo: la supuesta "naturaleza" maternal de las mujeres y la incompatibilidad entre ser mujer-madre-trabajadora.

Finalmente, a través de la pregunta de quiénes eran y qué hacían las mujeres y varones militantes del PRT-ERP y Montoneros pudimos reflexionar acerca de las permeabilidades e intersecciones entre los miembros de las organizaciones armadas y el 
contexto del que formaron parte. Es decir, que varones y mujeres militantes no se conformaron como subjetividades, ni como grupos aislados, sino que estuvieron fuertemente atravesados por aquellos procesos de sociabilidad de género que operaban en la sociedad que los vio nacer y de la que formaban parte. A modo de síntesis, observamos que, sobre un entramado altamente heterogéneo de situaciones, encontramos un péndulo que oscilaba diferenciando (hetero)sexualmente los espacios laborales de las y los militantes.

Desde un presente marcado por las movilizaciones feministas-disidentes, consideramos necesario continuar indagando no solo en la participación de las mujeres (y otrxs sujetxs) en los acontecimientos, sino también reflexionando críticamente acerca de los sexos y los géneros como construcciones sociales e históricas y no como hechos naturales. La incorporación de la perspectiva de género a la historia social del trabajo permite poner en diálogo las nociones estructurales respecto de la diferencia sexual en el mercado de trabajo -cuyas características exceden ampliamente los límites temporales aquí considerados- con las cuestiones coyunturales propias de la época como el proceso de industrialización y su impacto (o no) en las reconfiguraciones de la mano de obra masculina y femenina.

\section{Fuentes éditas}

Baracat, E. (1973). Situación de la mujer que trabaja, en la Provincia de Córdoba, Informe Secretaría Ministerio de Desarrollo de la Provincia de Córdoba.

Chamorro Greca, E. (1967a). La madre que sale a trabajar. En Memoria del VIII Curso de

Temporada. La mujer argentina y latinoamericana. Córdoba: Dirección General de Publicaciones, UNC.

Chamorro Greca, E. (1967b). La mujer en la estructura familiar. En Memoria del VIII Curso de Temporada. La mujer argentina y latinoamericana. Córdoba: Dirección General de Publicaciones, UNC.

INDEC. Censo Nacional de Población, 1960.

INDEC. Censo Nacional de Población, 1970.

\section{Referencias bibliográficas}

Águila, G, Luciani, L., Seminara, L. y Viano, C. (Comps.) (2018). La Historia reciente en argentina. Balances de una historiografía pionera en América Latina. Buenos Aires: Editorial Imago Mundi.

Andújar, A. (2017). Historia social del trabajo y género en la Argentina del siglo XX: balance y perspectivas. Revista Electrónica de Fuentes y Archivos, 8 (8), 43-59.

Andújar, A., D’Antonio, D., Domínguez, N., Grammático, K., Gil Lozano, F., Pita, V., Rodriguez, M. y Vassallo, A. (Comps.) (2005). Historia, género y política en los '70. Buenos Aires: Feminaria Editora. 
150 | Cartografías femeninas. Un acercamiento al mundo del trabajo...

Andújar, A.; D’Antonio, D.; Gil Lozano, F.; Grammático, K. y Rosa, M. (Comps.) (2009). De minifaldas, militancias y revoluciones. Exploraciones sobre los '70 en la Argentina. Buenos Aires: Ediciones Luxemburg.

Andújar, A., D’Antonio, D., Grammático, K. y Rosa, M. (Comps.) (2010). Hilvanando historias. Mujeres y política en el pasado reciente latinoamericano. Buenos Aires: Ediciones Luxemburg. [en línea: http://iiege.institutos.filo.uba.ar/otras publicaciones.php.]

Barragán Sáez, P. (2015). Mujeres trabajadoras y delegadas sindicales en un astillero de la Armada Argentina. Astillero Río Santiago (1973-1978). Nomadías, 20, 227-248 (URL: https://ri.conicet.gov.ar/bitstream/handle/11336/56710/CONICET_Digital_Nro.7b92 dbd7-a43a-4668-bfa2-2ee0b6c9df47_A.pdf?sequence=2\&isAllowed=y. (Descargado 15/03/2019).

Bohoslavsky, E. (Coord.) (2018). Debates y conflictos de la historia regional en la Argentina actual. Quinto Sol, 22 (3), 1-51. http://dx.doi.org/10.19137/qs.v22i3.3337

Brennan, J. (1996). El Cordobazo. Las guerras obreras en Córdoba 1955-1976. Buenos Aires: Sudamericana.

Cosse, I. (2010). Pareja, sexualidad y familia en los años sesenta. Buenos Aires: Siglo XXI Editores.

Cosse, I., Felliti, K. y Manzano, V. (Eds.) (2010). Los '60 de otra manera. Vida cotidiana, género y sexualidades en la Argentina. Buenos Aires: Prometeo.

D'Antonio, D. (2018). Bajas, cesantías, suspensiones y renuncias forzadas: trabajadores y trabajadoras del Estado en la mira (Argentina 1973-1983) (pp. 59-91). En D. D'Antonio (Comp.), Violencia, espionaje y represión estatal. Seis estudios de caso sobre el pasado reciente argentino. Buenos Aires: Imago Mundi.

D’Antonio, D. (Comp.) (2015). Deseo y represión. Sexualidad, género y Estado en la historia argentina reciente. Buenos Aires: Imago Mundi.

Di Liscia, M., Lasalle, A. y Lasalle, P. (2011). Verano del '72: La gran huelga salinera. Memorias, género y política. La Pampa: EdUNLPm.

Federici, S. (2010). Calibán y la bruja: mujeres, cuerpo y acumulación originaria. Madrid: Traficantes de Sueños.

Felitti, K. (2006). En defensa de la libertad sexual: discursos y acciones de feministas y homosexuales en los '70. Temas de Mujeres, 2 (2), 44-63.

Franco, M. y Levín, F. (2007). Historia Reciente. Perspectivas y desafíos para un campo en construcción. Buenos Aires: Paidós.

Fraser, N. (1997). Iustitia Interrupta. Reflexiones críticas desde la posición "postsocialista". Colombia: Siglo del Hombre.

Gatica, M. (2009). Industrialización y proletarización: las trabajadoras de INTECO en Trelew (pp. s/d). En E. Crespo y M. González (Comps.). Mujeres en palabras de mujeres. Rawson: Fondo Editorial Provincial.

Grammático, K. (2011). Mujeres Montoneras. Una historia de la Agrupación Evita. 1973-1974. Buenos Aires: Luxemburg.

Hall, C. (2013). La historia de Samuel y Jemima: Género y Cultura de la clase trabajadora en la Inglaterra del siglo XIX. Mora, 19 (2), 83-99.

Hartmann, H. (1980). Un matrimonio mal avenido: hacia una unión más progresiva entre marxismo y feminismo. Zona Abierta, 24, 80-105.

Jensen, S. (2010). Diálogos entre la historia local y la historia reciente en Argentina. Bahía Blanca durante la última dictadura militar (pp. 1426-1447). En XIV Encuentro de Latinoamericanistas Españoles: congreso internacional, Sep 2010, Santiago de Compostela, 
151 I Ana Laura Noguera

España. URL: https://halshs.archives-ouvertes.fr/halshs-00531187/document]. (Descarga 05/03/2019).

Laitano, G. y Nieto, A. (2019). 'Muñecas bravas en un nido de ratas'. Notas sobre las representaciones masculinas y el protagonismo femenino en las luchas gremiales de la industria del pescado. Revista Ejes de Economía y Sociedad, 4, 56-80.

Lobato, M. (2007). Historia de las trabajadoras en Argentina (1869-1960). Buenos Aires: Edhasa.

Manzano, V. (2018). La era de la juventud en Argentina. Cultura, política y sexualidad desde Perón hasta Videla. Buenos Aires: FCE.

Martínez, P. (2009). Género, política y revolución en los años setenta. Las mujeres del PRTERP. Buenos Aires: Imago Mundi.

Mitidieri, G. (2014). La huelga de Alpargatas de 1979: las nociones de lo justo en la dictadura. Revista Páginas, 6 (12), 83-102.

Noguera, A. (2019). Revoltosas y revolucionarias. Mujeres y militancia en la Córdoba setentista. Córdoba: Editorial de la Universidad Nacional de Córdoba.

Oberti, A. (2015). Las revolucionarias. Militancia, vida cotidiana y afectividad en los setenta. Buenos Aires: Edhasa.

Ortiz, L. (2019). Con los vientos del Cordobazo. Los trabajadores clasistas en tiempos de violencia y represión. Córdoba: Editorial de la Universidad Nacional de Córdoba.

Partenio, F. (2017). Género, trabajo y experiencia: perspectivas teórico-metodológicas para el abordaje de las narrativas biográficas (pp. 81-97). En K. Grammático, M. Marini y W. Wechsler (Comps.), Historia Reciente, género y clase trabajadora. Buenos Aires: Imago Mundi.

Pasquali, L. (2008). Mandatos y voluntades: aspectos de la militancia de mujeres en la guerrilla. Temas de Mujeres, 4 (4), 49-75.

Pateman, C. (1995). El contrato sexual. Barcelona: Anthropos.

Pérez, I. (2010). El trabajo doméstico y la mecanización del hogar: Discursos, experiencias, representaciones. Mar del Plata en los años sesenta (pp. 171-204). En I. Cosse, K. Felitti y V. Manzano (Eds.), Los '60 de otra manera. Vida cotidiana, género y sexualidades en la Argentina. Buenos Aires: Prometeo.

Recchini de Lattes, Z. (1980). La participación económica femenina en la Argentina desde la segunda posguerra hasta 1970. Buenos Aires: CENEP.

Romano, S. (Ed.) (2016). Colectivos y parcialidades políticas y sociales: los desaparecidos y asesinados de Córdoba en los'70. Córdoba: Editorial FFyH-UNC.

Scott, J. (2011 [1999]). Género e Historia. México: FCE.

Sepúlveda, P. (2015). Mujeres insurrectas: condición femenina y militancia en los '70. Buenos Aires: Universidad Nacional de Quilmes.

Thompson, E. P. (1989). La formación de la clase obrera en Inglaterra. Barcelona: Crítica.

Viano, C. (2011). Pinceladas sobre las relaciones de género en la nueva izquierda peronista de los primeros años '70. Temas de Mujeres, 7, 233-252. 
152 | Cartografías femeninas. Un acercamiento al mundo del trabajo...

Para citar este artículo:

Noguera, Ana Laura (2019). Cartografías femeninas. Un acercamiento al mundo del trabajo con perspectiva de género. Córdoba, 1966-1976. Anuario de la Escuela de Historia Virtual, 16, pp. 137-152. 